\title{
A Basic Research for the Holographic Creation for the Utilizing Visualization
}

\author{
Keejung Barng ${ }^{1}$ \\ ${ }^{1}$ Dept. of Beauty \& Health Management, Daejeon University \\ 62, Daehak-ro, Dong-gu, Daejeon, 34520, Korea \\ wp2848@nate.com
}

\begin{abstract}
This study utilized a digital visualization tools with holographic creation basic research. This study utilizes a hologram to reproduce and how traditional Korean makeup how to apply. This study is a Korean traditional makeup base for effectively communicating research. Holographic takes advantage of digital video media to apply. Traditional Korean costumes, props, make-up and how to dress, hair ornament, holographic representation. For more information about how the makeup is common in light-colored holographic, saturated, vivid tones effectively. Much of the movement in the holographic model gave the movement, the effect of dust, vibration screen. And many difficult movements corrected. However, the hair color, the size and shape of the head of the black reflecting is expressed in the form. Minutes from great reflective holographic facial color is black or transparent. Reflection and diffraction of light skin representation of the model using the principles apply. Minute chapter skin and skin color Eclipse much of the mat as an expression. What is the color of the skin, face, and white or bright makeup is in the form of a three-dimensional face and noticeably. I call the holographic representation of the chapter in order to light, wind, etc., influenced by the degradation of the surrounding factors and the screen resolution; the screen can be easily shaken. I call the holographic representation of the chapter in order to vibration and interference and diffraction of light and color wavelength using consideration should select a color.
\end{abstract}

Keywords: Holographic, Digital Image, Traditional Makeup, Korean Makeup, Digital Makeup

\section{Introduction}

The Knowledge of the information age of the 21st century in the field of new technologies and the right social information each day has been pouring in. A new expression to fit the flow of the society in the field of imaging techniques and technologies are introduced. These rapid changes are varied and wide range of expression video making. There are also state-of-the-art tools and equipment for video technology is required (J. H. Yun, 2007). While entering the digital age technology is developing very fast. Digital is a fusion of different disciplines and has become very rich and complex. These changes in the social and cultural environment, advances in technology, we close in life, such as social, cultural, lifestyle, and environment of humanity is evolving. Through the Internet we are temporal, spatial constraints beyond anywhere, anytime access. We have a wide variety of content and information in real time to quickly update. There is also a wide variety of skills and technology, melting, complex features a variety of functions as a result of the fusion of the complex trends, and the design also has been a big change (K. J. Barng, 2012). The complexity of the development of science and technology in the digital age is changing the creation of a distinctive character to create a

Received (December 7, 2017), Review Result (February 10, 2018), Accepted (February 15, 2018) 
hybrid character. These are the types of animal and human hybrid heterogeneous coupling, plants and human hybrid, cyborg and human hybrid, based on the surreal character who won last name (Y. K. Kim, S.Y. Shin, 2014). The beauty of Korea has attracted attention to the growth of Korean child care and K-Pop singer of the market. Beauty and makeup how traditional methods of Korea have attracted attention. There is also a variety of cultural and social leverage through the beauty of South Korea K-Beauty. Recently a lot of the traditional form of a new fusion attempt takes place. Among them, the most innovative example is the meeting of traditional and cutting-edge technology. To preserve the original appearance of traditional culture and the aesthetic value and the technical values are passed down culturally meaningful. This can be seen high value makes the traditional arts and traditional values and state-of-the-art digital technology, the convenience of daily life has a different meaning to each other (D. Y. Lee, 2010). In the anonymity of the digital culture, mutuality, the characteristics of the immediacy, a bit like in the real world do not feel even the impact on users ' sensibilities. Depending on the change of era showcase video communication system in the presence of the media evolved way of display needs was transformed into an exhibit. Through the development of display media reinforces and enhances the quality of the visitors and of communication Interaction is not aware of this new role is important. Humans will never experience the perfect gods envy sometimes for dreaming. Experience this desire to solve the human longing to be able to defraud the world of reality, not a virtual world. In reality, due to the physical constraints of the spatial, may indirectly experience the experience. In this way, one of the most important factors is the reality of virtual reality and real-life Visual representation of the elements, connecting interface. In other words, these virtual and real realities that connect the interface display technology. The holographic principle and outstanding due to its characteristics, such as reproducibility and Zhejiang Province are being noted by a futuristic 3D stereoscopic video. Interactive and can communicate with the world in a format that virtual reality is becoming instrumental in the promising field of representation (T. K. Kim, 2010). In the study, followed by relevant to Bang (2015) can take advantage of the convergence of three dimensional simulation program developed and proposals mentioned in the. However, due to the efficient representation of digital tools and take advantage of technologies applied research on how is still poor. In this study, how to leverage the tools and makeup changes centered on the convergence of digital technology and content-centered. A more macro perspective, compare in terms of socio-cultural, physical tools will see in this study, a case to apply the holographic representation of the way through the future digital media and would like to offer a guide line for the efficiency that is the purpose of this study. The goal of this research for holographic arts sector, using, inter alia, through the effective methods for the study of the convergence of broadcasting video for digital and physical methods should you wish to explore. This research takes advantage of the digital content convergence and for the development of complex research. This study, culture, art and technology to reproduce a great visualization I call the color, texture and form and in the category of dress on the convergence of digital holographic technology and technique-oriented. Most of the art and cutting edge technology in the field of stage and beauty industry a fusion of digital technology to take advantage of the efficiency of the holographic through and know about. So this study, Korean makeup how to effectively take advantage of digital video media for passing. Digital image holographic video production by applying to the Foundation for research, this is the purpose of this study. Holographic takes advantage of digital video media coverage, the traditional Korean costumes, props, make-up and how to dress, hair ornament, holographic representation. 


\section{Research Methods}

This study is a Korean traditional makeup to effectively take advantage of digital video media for passing. Digital Imaging is the basis for the creation of the holographic application and research in order to proceed to the following method.

\subsection{Research Issues}

1) This study is a literature research and leading research holographic note is the digital display technology is the basis for research.

2) In this study, work practices, the most efficient digital visualization can be represented as a holographic way.

3) Among the artifacts, exhibitions and galleries of digital display technology, rather than moving to take advantage of the holographic video practices via basic research.

4) In this study, a special figure in the Joseon dynasty to relive the best and, line, form, color holographic visualization by comparing the basic research was

\subsection{Materials and Data Collection}

Research methods literature research, Internet navigation, was composed by way of case studies. A special figure in the Joseon dynasty to relive the best and, line, form, color holographic visualization by comparing the basic research was. For more information about how the era of Korean traditional makeup, portrait, welcome reproduced through the literature about how to configure how the contents of the.

\subsection{Measuring Tools}

First, I reproduced the traditional Korean makeup is a Joseon Dynasty centered on oath law literature makeup the best and leading research note said the clean-up. Korean makeup how to apply a technique called digital hologram figure of Joseon Dynasty Hwang Jin Yi. Selected as one of the reasons is one of the most colorful figures in the best selected and unique makeup did have a parasite called identity. It also shows a special layer of identity by dance and art, which is the chapter minute conversation examples, including that of the artistic part of the show is a portrait. Second, the theoretical background for literature research holographic make sure you would actually find them specific examples that have been cleaned up. And, based on this solid dark black space at the hologram are shown the best way to show the best makeup colors, lines and shapes, the clothes, jewellery, accessories, hair, such as the shape and form.

\subsubsection{Holographic}

Holographic word meaning all, entire, complete with the ' $\mathrm{H}$ o $1 \mathrm{o}$ ' and means ' a picture, AutoShape, G r a p h photos i c s is a portmanteau of '. Did you mean complete picture of holographic stereoscopic video which says (Han, 2001). The holographic characteristics of the concept the term was defined. And Korean traditional makeup and apply the law related to the analysis and definition of the term through their operational definitions.

\subsubsection{Hologram}

Hologram is $\mathrm{Ho} \mathrm{Gra} \mathrm{mol'}$ ' and ' $\mathrm{e}$ ' is a portmanteau of. Etymology of the word in Greek means ' $\mathrm{H}$ o o $\mathrm{l} \mathrm{s}$ the totality ' and recorded, message, information means ' $\mathrm{G} \mathrm{r}$ a $\mathrm{m}$ a ' will come from. The hologram is an object which has a complete system of the full record information (Kim, S. C., 2006). 


\section{Theoretical Background}

What is disappeared come back to reproduce Dunn festival performed in the old things still look back to the past or the old palace says that progress. Reproduction can be described as "restoration of the facts or recurrence '(Hong, 2016). Reproduction can be described as "restoration of the facts or recurrence '(Hong, 2016). Hologram technology refers to the human-friendly realistic three-dimensional image that provides the same look as realistic as real objects (Cho, Alliance, 2015).

\subsection{Holographic}

We usually have a 2-d plane photo film camera or digital camera to shoot through a general photography. In other words, the contrast of light and color in the target object of two-dimensional information means that you can record. But holography using stereoscopic video light waves (frequency, phase, and amplitude), through the interference of the Wave front 3D information to record and play. Holographic laser is using the characteristics of an object reflected waves from other objects, expand the basis of the principles of optical waves straight to holography. This means that each object in the sector by the State interferes with information one by looking at the substance of the film, leaving no stone unturned in the record, such as stereoscopic Imaging. 3D stereoscopic images of-the-art optical technology to reproduce these photos as a transparent Crystal technique, using a stereoscopic 3D video. Holographic display of cultural heritage using cultural heritage intact can reproduce the real-world. So the hologram is used a lot as a value in a museum. Holographic video that plays as accurately and precisely, even a microscopic part of the recorded images even when enlarged you can observe express perceptual effect (Cho, J. H. \& Jo, J. H., 2010). A wing of a butterfly has shown the symptoms of the indirect light due to the thin film. Throw a stone in a calm lake, rock while drawing concentric circles of waves are ripples around the missing points can be seen that the outgoing transfer outside. All this has the wave of specific amplitude and the oscillation number of the phase. It is well known that the nature of the light of a kind of electromagnetic waves. Light is also creating an interference pattern of darker or lighter or by interference like any other wave.

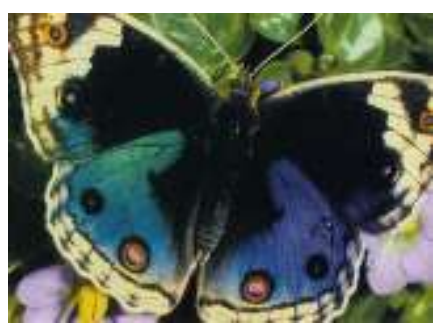

Figure 1. Interference of Light - a Butterfly Wing is Interference of Light by Thin Film

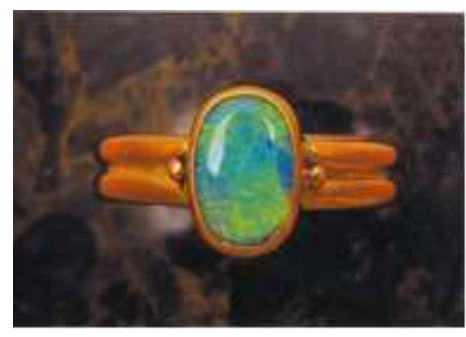

Figure 2. Diffraction of Light Jewelry: the Diffraction of light, such as Opal

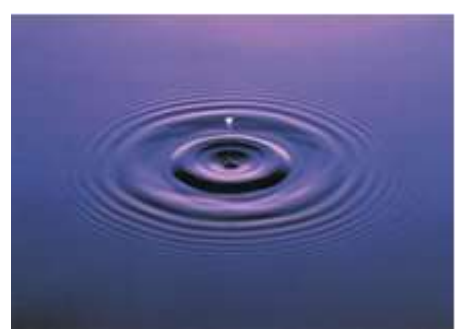

Figure 3. The Ripples of the Water

\subsection{Hologram}

The hologram is a compound word of 'Holo' and 'Grame' origin is recorded as 'Holos' which means Greek perfection, and record the complete whole message, the hologram to come from the 'Grama', meaning information has the object means that the information (Kim, SC, 2006). The hologram is a technique for describing the objects to the 3D display without the use of coherent light. Reflective light on the real thing, or recall the record 
comes out distribution and reproduction. Therefore, the implementation can be divided into a reflection type, transmission type, the rainbow-type. The hologram is classified into analog and digital hologram. There are similar hologram holographic images that mimic the effects. Analog hologram is a technology that picture through the art of using the film to take a real three-dimensional image of the target object, such as reproduction of the real thing. Similar hologram is a technology for implementing an effect similar to the semitransparent screen image, a second holographic three-dimensional image point again. Is a technique for digital hologram recording and reproduction through the digitization of the light reflected from the object, such as the actual service realism (Cho, Alliance, 2015). Is a technique for digital hologram recording and reproduction through the digitization of the light reflected from the object, such as the actual service realism (Cho, Alliance, 2015). Holography is able to record the location and status information of the object by the interference of two light waves and of Reference Object wave. It is written in such a way that the film of the photo, Hologram. Here again is to reproduce three-dimensional video of shining light. This video is the location of the target object normal photos and play with the State's information. In particular, stereoscopic 3D to implement two dimensional images and the video is a tremendous amount of information that should not be a comparison is needed. This export it is reproduced by receiving real-time current information processing technology, transmission technology is hardly impossible (Cho, J. H. \& Jo, J. H., 2010). Current technologies such as floating scheme for performances illuminates a mirror on the floor, like a kind of hologram and a transparent film by reflecting the image of the object as a kind of illusion (Cho, Alliance, 2015). If the hologram is fully implemented, but to see the gun 3D images from any angle and is not yet commercially step.

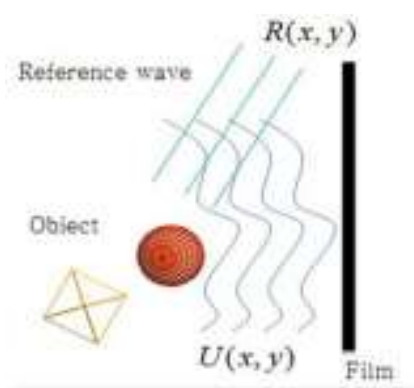

Figure 4. Recording and Reproduction of a Hologram

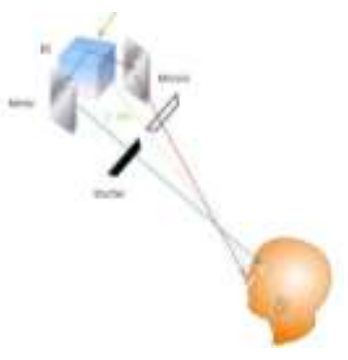

Figure 5. Time-Sharing System Configuration

The principle of alone Photography is in pointing the subject with a light beam divided into two by the coherent light from the laser light source beam splitter, of which the subject surface I reflection light reaches alone Photography photosensitive material do. This beam is called the object beam. The other light beam is lit in the front hall directly Photography photosensitive material diffuses into the lens. This is called a reference light beam. If this Holographic photosensitive material object beam and the reference light interfere with each other $500 \sim 1,500$ per $1 \mathrm{~mm}$ raise the level of development makes very delicate and complicated interference patterns. Photos recorded interference pattern is called g alone. Chroma-key technique is the blue bag (back) and blue of the subject photographed as part of the General, but not necessarily to burn synthetic Blue does not mean you need to be. Usually the Key (with subtitles or a specific type of signal) acts as the difference between the black and white video signals but separated by a difference of colors Chroma-key Key to the behavior of the technique. Select any specific color to create a key signal. Currently, the Chroma-key Edition of blue or green, place the camera person or object in front of a shot by blue or green portion of the difference in the picture will replace the Chroma-key. Green or red too synthetic, but why use blue Carnation 
because it is the complement of a human, without affecting the synthetic, because blue. The structure of one particular color Chroma-key (blue) and divided into a non-color, specific colors (blue, green) that any other background image synthesis on your part.

\section{Result and Discussion}

3D hologram 360 is a technology for implementing a three-dimensional image can be seen in all directions. 3D stereoscopic image generated in 3D hologram technology has an advantage to enhance the realism and immersion by providing a three-dimensional appearance, such as if the original. Hologram records the distribution of the light that is reflected or diffracted from the actual radio wave by means seeing the overall appearance of the object is reproduced. Holograms are fully implemented in the real image that appears three-dimensional space (Cho, Alliance, 2015). Holographic display of cultural heritage using cultural heritage intact can reproduce the real-world. So the hologram is worth as much as a high use of Cali at the Museum. Holographic video that plays as accurately and precisely, even a microscopic part of the recorded images even when enlarged you can observe express perceptual effect (Cho, J. H. \& Jo, J. H., 2010). This study is an effective way to deliver the traditional Korean makeup for fusion research. Take advantage of the digital video images, which is why the media is accurate and vivid use of holographic. Apply via the digital holographic video and for basic research in order to proceed to the following method. In this study, Korean makeup how to apply a digital three-dimensional holography and basic research for the creation for research as follows. This study has been applied for the scenario used the holographic Joseon Dynasty had run a special status with the squad the best makeup for makeup how to proceed. Select the dialog sheets a minute through Hwang Jin-Yi Joseon Dynasty why Hwang Jin-Yi was a very special person called. The best of the Joseon dynasty is a special identity is referred to as courtesans with art. She is very gorgeous, colorful ornaments in a variety of outfits and was using a hierarchy. Since the background is black, colorful costumes and graphics alone, ornaments, and to reproduce vivid makeup and special Hwang Jin Yi is best suited. 'Day of the Gyo-bang ' bring up the subject, known as stereoscopic images. Gyo-bang's doors open, since it is the best of a mediocre day be parasitic. The door opens the banquet of the Gyo-bang Joseon dynasty to prepare for the appearance of a woman. Hwang Jin Yi, head of the tax revenues to Foundation makeup. Hwang Jin Yi in the face is smooth hair, rubbing white minutes. And, the best makeup in the guise of a squad finished a gorgeous ornaments and costume. Computer graphics transformed the appearance of the video through staged through the best. Hwang Jin Yi is dancing and playing the harp. Finally, finish the look through the eponymous 180 seconds video.
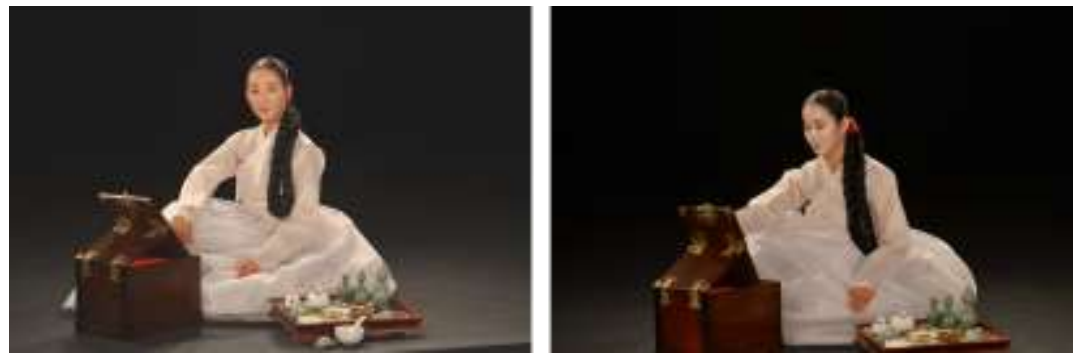

Figure 6. The Basic Makeup and Hair Theorem 

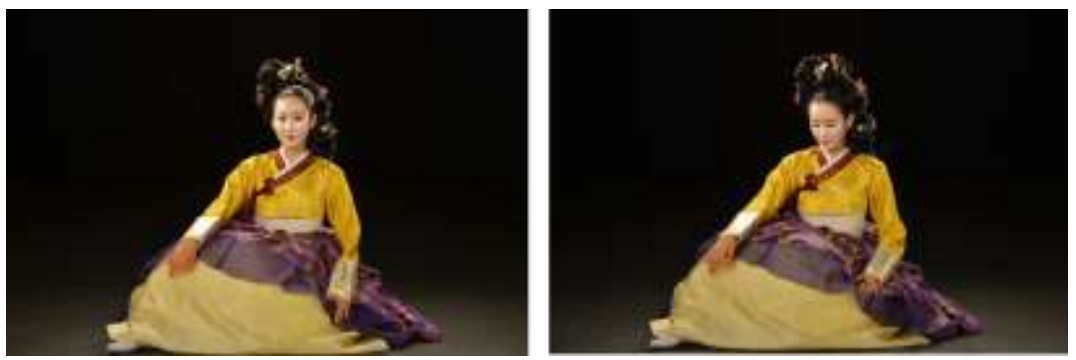

Figure 7. Bundle Makeup and Costume
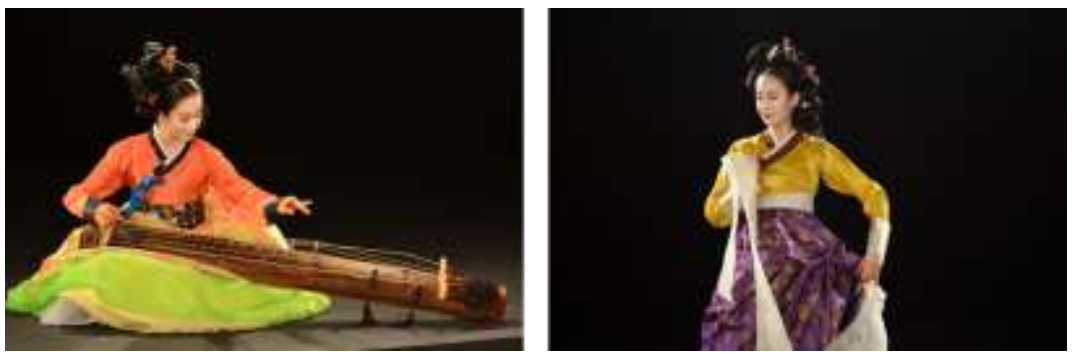

Figure 8. Hwang Jin Yi played a Lyre and Dance

This enables holographic 3D stereoscopic video production for producing holographic for application and content are as follows: Hwang Jin Yi costumes, props and ornaments used in contrast with the background on a black stage, a light color. Color is white or light-colored, and saturation, and brightness, crisp vivid tones (Vivid Tone) was very well represented. In addition, it is transparent, permeable forms an invisible black, Brown, blue-green, blue color, dark color, low color saturation, low-brightness low dark color are avoided or not. What is the color of bright yellow, Orange, Chartreuse, such as colors, the family chose. In addition, you can reactivate the shape overlap wrinkles or the costume colors via the embedded phenomenon to a minimum. Clothes in the end of a line, the pus or skirts tied white by placing a color control. All the best costumes and props and even as much as possible in order to revive the form lines and bright costumes look pleated are appropriately applied.

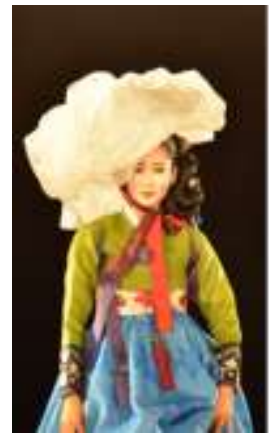

Figure 9. Holographic Costume and Production
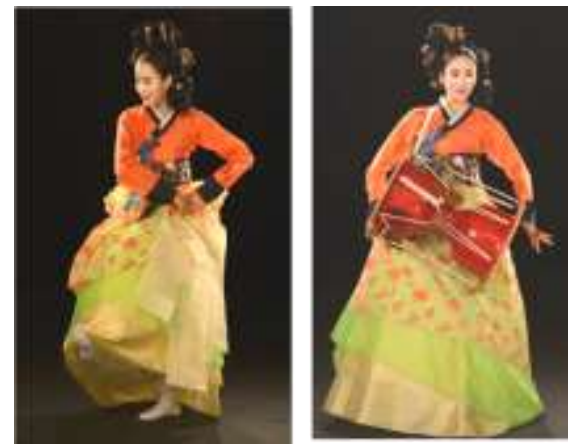

Figure 10. Holographic Costuming and Props and Production

Use these colors in here that even if dynamic parts of the static part of an object, which can be displayed. In addition to the movement of a dynamic part of the motion model and vibration, such as dust, giant screen, the screen calibration as a base to Paris. There is also a static part of the props, and so there is no vibration or movement of movements because they are not after the shooting to complement the color correction was able to work with color. 

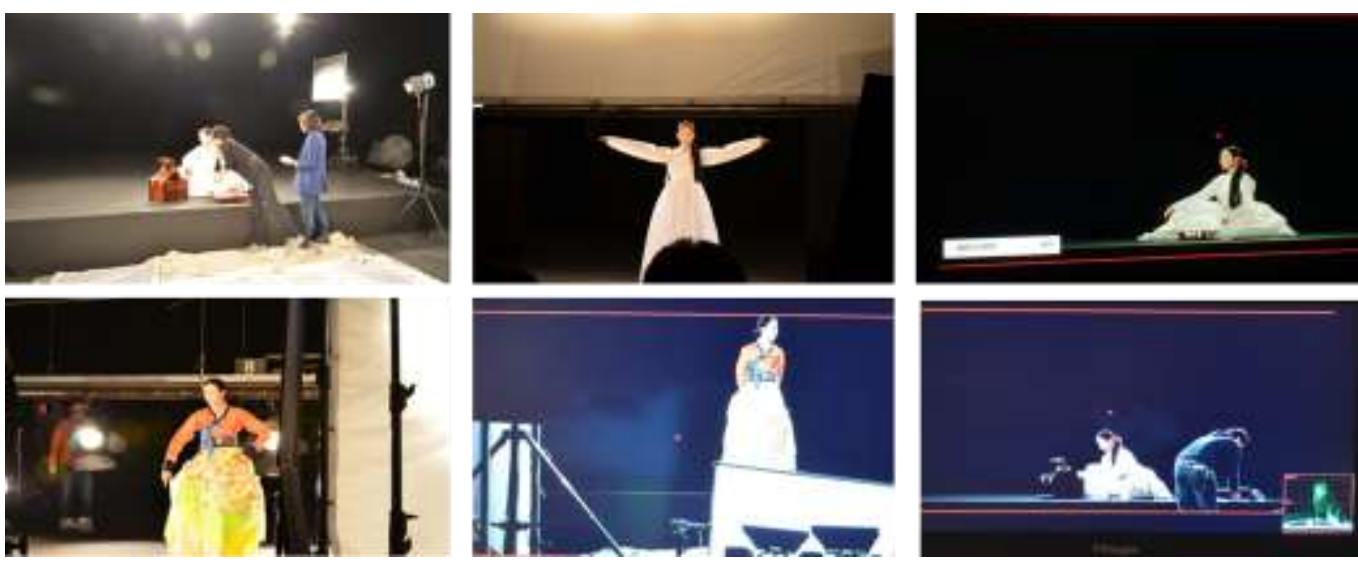

Figure 11. Apply the Holographic Configuration of the Timeshare System
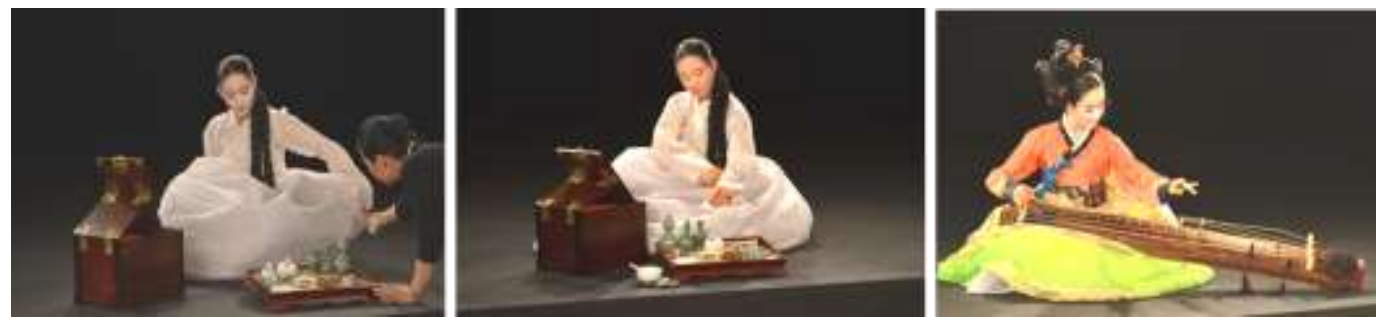

Figure 12. Static/Dynamic Holographic Costuming and Props and Production

Hair and am using a black light in contrast with the background stage of great colors. In particular, white, bright color, and saturation, and brightness, vivid color is expressed very well. Light colors are transparent, permeable formations by invisible black color, dark-colored, low-saturation, brightness, color or do not use. However, the color of the hair, black hair, but light colors reflected the colorful hair, such as the classic head of black color, shape and form and size has been observed. So, the boundaries of the head and the point of contact on the part of the colorful non-transparent and various trinkets, by taking advantage of the shape of the head was shown. Such a gorgeous head of hair, twist, etc. and the size of the classic head were shown in the form. The background lighting of the stage is very strong; the Chairman of minutes black, the reflection of light because the media using the principles of facial skin is extremely glitter seemed to. The face is black and shiny parts of the face, not being transparent, despite setting of the forms look like. A holographic representation of the light reflection and diffraction of the skin for the video using the principle expressed in the skin, skin color is matte, thick due to Eclipse much of the face expression. The holograms should be white and bright makeup face in the form of a three-dimensional face and showed significantly. In order to play on stage color hologram multi-wavelength light source should be used. However, the optical system by using a hologram when you want to play, color separation happens; the quality of the image to play the quality is a factor in the drop. So in this study, characteristics of holographic imagery centered on the substance of the wavelength of light to play with the colors, the color information to the extent that the amplitude of light and dark. And, for playing the videos brightness, texture and form a sense of depth as an expression of phase with a focus on production. 

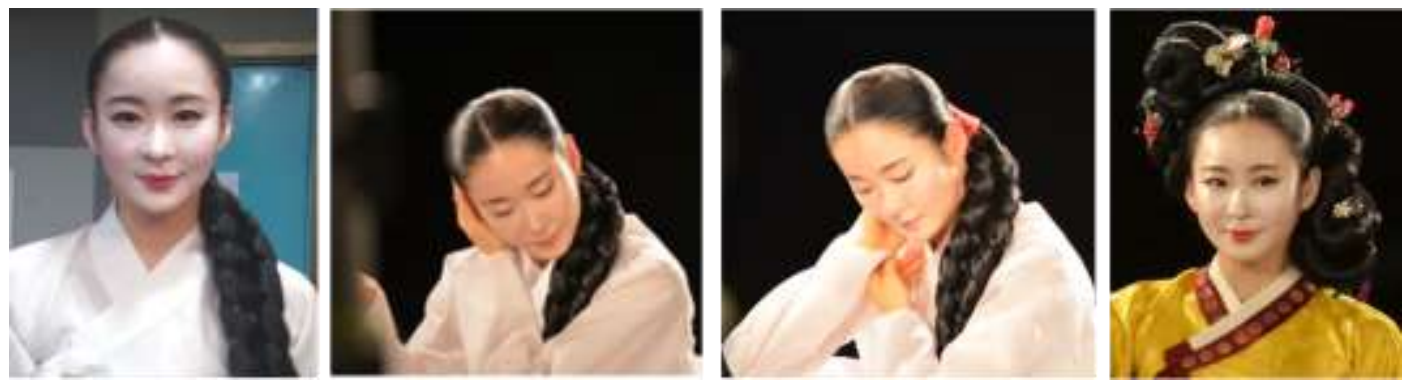

Figure 13. Holographic Dress and Hair and Production

How holographic 3D stereoscopic video production Joseon dynasty beauty holographic applied for and progress for producing summary tables as follows.

Table 1. Joseon Dynasty Makeover of the Act Applies and Makes the Holographic Process Digital Video

\begin{tabular}{|c|c|c|c|c|}
\hline & & Color and shape & Expressed Features & $\begin{array}{l}\text { Production } \\
\text { information }\end{array}$ \\
\hline $\begin{array}{l}\mathbf{C} \\
\mathbf{O} \\
\mathbf{S} \\
\mathbf{T} \\
\mathbf{U} \\
\mathbf{M} \\
\mathbf{E}\end{array}$ & $\begin{array}{l}\mathbf{G} \\
\mathbf{O} \\
\mathbf{O} \\
\mathbf{D}\end{array}$ & $\begin{array}{l}\text { - Reflection and } \\
\text { diffraction of light } \\
\text { - White and red colors } \\
\text { - High saturation, } \\
\text { lightness color } \\
\text { - Vivid Tone } \\
\text { - Black } \\
\text { - Dark } \\
\text { - Low saturation, also } \\
\text { prominently black } \\
\text { - Thick } \\
\text { eyebrows }\end{array}$ & $\begin{array}{l}\text { - Bright colors series } \\
\text { - Yellow, orange, light green, etc. } \\
\text { - Costumes induced wrinkles or } \\
\text { overlapping forms possible } \\
\text { - This phenomenon leads to a } \\
\text { minimum through color invisible. } \\
\text { - White color controls placed on } \\
\text { clothes pus, line skirt, jacket } \\
\text { sleeve. } \\
\text { - Properly apply the light color } \\
\text { lines and wrinkles. }\end{array}$ & \\
\hline $\begin{array}{l}\text { A } \\
\text { C } \\
\text { C } \\
\text { E } \\
\text { S } \\
\text { S } \\
\text { O } \\
\text { R } \\
\text { I }\end{array}$ & $\begin{array}{l}\mathbf{G} \\
\mathbf{O} \\
\mathbf{O} \\
\mathbf{D}\end{array}$ & $\begin{array}{l}\text { - Reflection and } \\
\text { diffraction of light } \\
\text { - White and red colors } \\
\text { - High saturation, } \\
\text { lightness color } \\
\text { - Vivid Tone }\end{array}$ & \begin{tabular}{l|l} 
D & The movement of the model, \\
$\mathbf{Y}$ & such as dust, vibration is a \\
$\mathbf{N}$ & large point on the screen the \\
A show in Paris, and the \\
M calibration is difficult. \\
I - Select the location of the \\
C model's position and \\
movement within the specified \\
area is minimized
\end{tabular} & \\
\hline $\begin{array}{l}\mathbf{\&} \\
\mathbf{J} \\
\mathbf{E} \\
\mathbf{W} \\
\mathbf{E} \\
\mathbf{L} \\
\mathbf{R} \\
\mathbf{y}\end{array}$ & $\begin{array}{l}\mathbf{B} \\
\mathbf{A} \\
\mathbf{D}\end{array}$ & $\begin{array}{l}\text { - Black, brown, } \\
\text { navy blue, blue, etc. } \\
\text { - Low saturation, even } \\
\text { prominent dark }\end{array}$ & 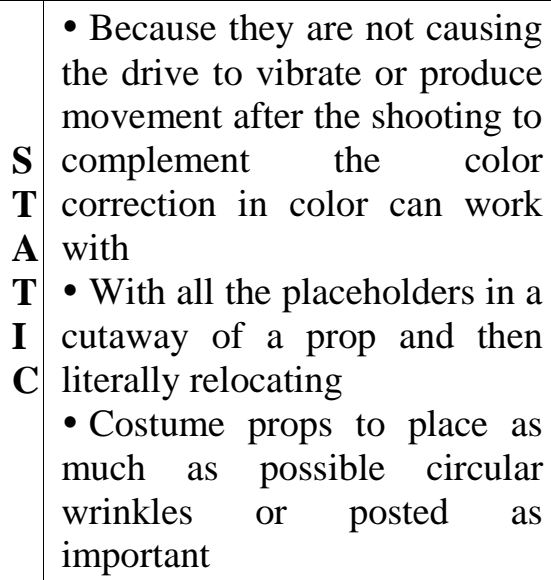 & \\
\hline
\end{tabular}




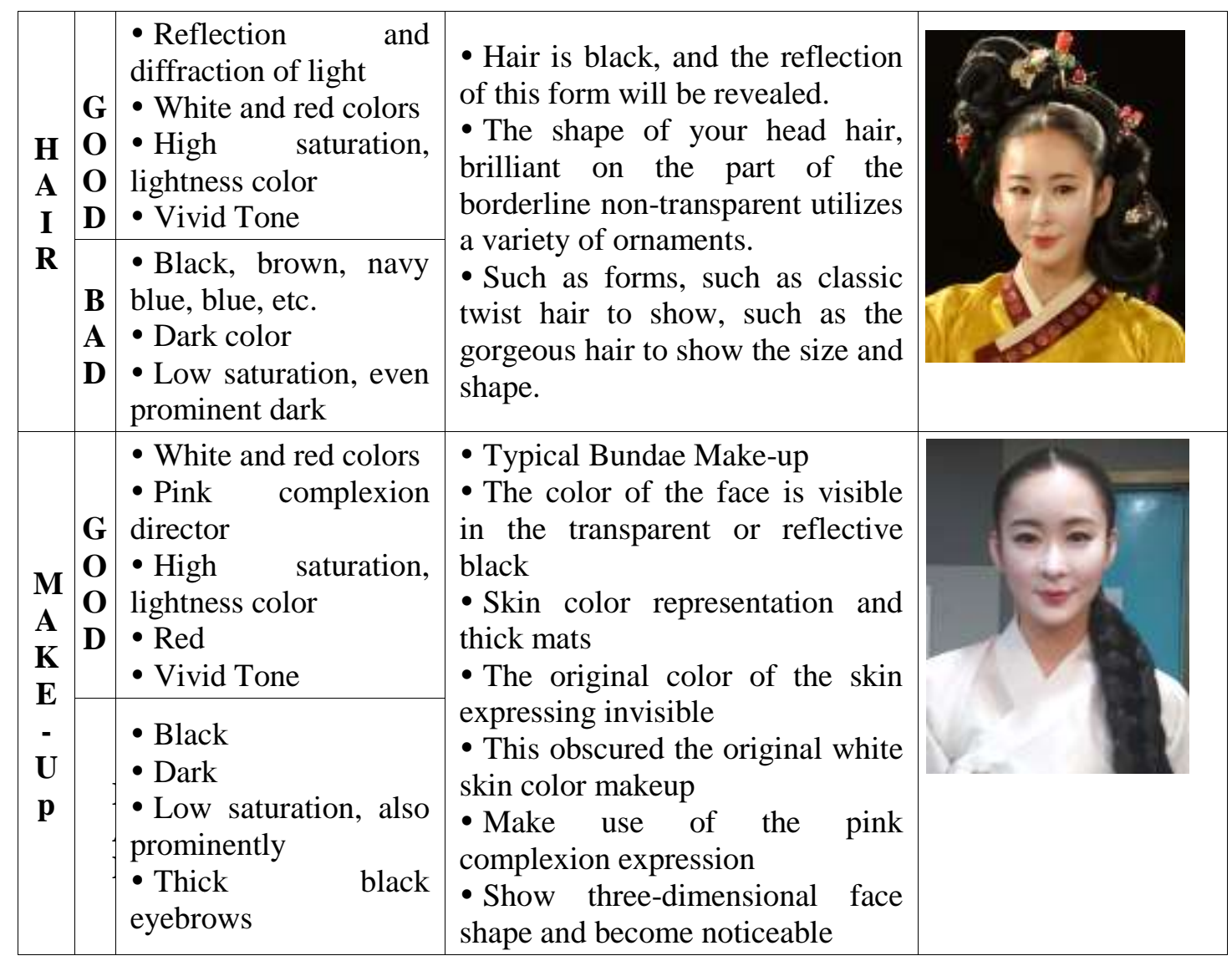

\section{Conclusions}

This study is a Korean traditional makeup to effectively take advantage of digital video media solutions for delivering digital video applied via holographic and is the basis for the creation of research for the purposes of this study, you would want to. Hwang Jin Yi costumes, props and ornaments used in contrast with the background on a black stage, a light color. Color is white or light-colored, and a saturation, and brightness, crisp vivid tone was very well represented. In addition, it is transparent, permeable forms an invisible black, Brown, blue-green, blue color, dark color, low color saturation, low-brightness low dark color are avoided or not. What is the color of bright yellow, Orange, Chartreuse, such as colors, the family chose. In addition, you can reactivate the shape overlap wrinkles or the costume colors via the embedded phenomenon to a minimum. Clothes in the end of a line, the pus or skirts tied white by placing a color control. All the best costumes and props and even as much as possible in order to revive the form lines and bright costumes look pleated are appropriately applied. Use these colors in here that even if dynamic parts of the static part of an object, which can be displayed. In addition to the movement of a dynamic part of the motion model and vibration, such as dust, giant screen, the screen calibration as a base to Paris. There is also a static part of the props, and so there is no vibration or movement of movements because they are not after the shooting to complement the color correction was able to work with color. Hair and am using a black light in contrast with the background stage of great colors. In particular, white, bright color, and saturation, and brightness, vivid color is expressed very well. Light colors are transparent, permeable formations by invisible black color, dark-colored, low-saturation, brightness, color or do not use. However, the color of the hair, black hair, but light colors reflected the colorful hair, such as the classic head of black color, shape and form and size has been observed. So, the boundaries of the head and the point of contact on the part of the colorful non-transparent and various trinkets, by taking advantage of the shape of the 
head was shown. Such a gorgeous head of hair, twist, etc. and the size of the classic head were shown in the form. The background lighting of the stage is very strong; the Chairman of minutes black, the reflection of light because the media using the principles of facial skin is extremely glitter seemed to. The face is black and shiny parts of the face, not being transparent, despite setting of the forms look like. A holographic representation of the light reflection and diffraction of the skin for the video using the principle expressed in the skin, skin color is matte, thick due to Eclipse much of the face expression. The holograms should be white and bright makeup face in the form of a threedimensional face and showed significantly. Holographic is the constant research and development of technology in a variety of natural color representation as possible. Holographic representation of a non-stationary and moving images and video footage was possible, such a dazzling performance, but a complete virtual reality is still insufficient. It is difficult to use in a bright place, holographic, let the air out of the light reflection on the impact of wind or vibration, such as waking is easily shaken by the degradation of factor can cause the resolution can be. So the various elements of the crisp, clear color by implementation remains an issue. I call the holographic representation of the chapter in order to light, wind, etc., influenced by the degradation of the surrounding factors and the screen resolution; the screen can be easily shaken. In order to apply the holographic principle of vibration and interference and diffraction using costumes, props and jewelry, dress and hair color for the wavelength of the light and color should be chosen. Video media takes place in a variety of areas to take advantage of. For more information about how the novelty of using traditional Korean makeup note rate to show a hologram and attractive for the media could increase suitable as a medium I think worth researching. In this study, various exhibitions and fairs in recent major advertising or an up-and-coming video medium such as hologram methodology through the utilization of Korean traditional makeup and basic research to take advantage of as a resource. For more information about how the traditional Korean makeup cases are scarce mostly 2D TV documentary format through domestic research. However, this research is the application of stereoscopic 3D hologram limited and empirical research for the Foundation for providing the material means to this study. In this study the 2013 World Expo theme pavilions O-song cosmetics and beauty world beauty from the exhibit display to use as a video production. How to exhibit traditional Korean makeup video presented through a video production course for holograms. The holographic configuration of a digital display, and content through a holographic 3D stereoscopic video production presented by the Foundation for data, can help you become a boon to the objective data. In a subsequent study, these findings provided the Foundation for the theory, based on continuous observation and in-depth research is needed. Also take advantage of a variety of digital content creation and will require a specific study on the production of animal feed.

\section{References}

[1] K. J. Barng, "Korean Traditional Make-up of the Act Applies and the Holo-graphic Digital Video Production for Basic Research", Asia-pacific Proceedings of Applied Science and Engineering for Better Human Life, vol.10, (2016), pp. 97-100

[2] Y. Y. Han, "Study on a 3D stereoscopic production techniques", Unpublished master's thesis, Hongek University, Seoul, (2001).

[3] H. J. Kim, "research for the communication effect of hologram technique in outdoor advertising", unpublished master's thesis, Hongek University, Seoul, (2002).

[4] T. K. Kim, “3D Display Holographic 3D imaging system views”, Optical science and technology, vol. 14, no. 4, (2010), pp. 30-34.

[5] S. C. Kim, "Implementation of Computer-Generated Holograms of 3D Objects Using Depth Information", Unpublished master's thesis, Kwangwoon University, Seoul, (2006).

[6] D. Y. Lee, "The Aspects of Technological Response on the Mode of the Performing Arts of Tradition", Journal of the society for Korean historico-musicology, vol. 44, (2010), pp. 325-336.

[7] J. H. Cho and J. H. Jo, "A Study of the Potential of Next Generation 3D Interactive Video Media Via Holographic", Journal of the Korean Society of Design Culture, vol. 16, no. 2, (2010), pp.436-448. 
[8] Y. J. Joe, “A Study on Visual Perception of Hologram Advertisement”, the Korea contents Society, vol. 6, no. 12, (2006), pp. 261-268.

[9] J. H. Yun, "Simulation of Efficient Virtual Space using 3D Holography", Unpublished master's thesis, Hanyang University, Seoul, (2007).

[10] K. J. Barng, "Makeup Design and the Application of 3D Facial Avatar Makeup Simulation”, Journal of fashion business, (2014).

[11] K. J. Barng, "3D Facial Avatar Make Up Simulation) Program development (Vol 1)",Journal of the Korean society of cosmetology, vol. 21, no. 2, (2015), pp. 1-9

[12] K. J. Barng, "TV documentary on the Joseon Dynasty use employment law analysis; Jae Sung-centered theory parameters", Journal of fashion business, vol. 19, no. 5, (2015), pp.48-62

[13] J. S. Hong, "TV Studio production practice", Seoul; Communication Books, (2016), p.12

[14] K. J. Barng and J. S. Kim, "the development and utilization of special makeup mask 3D simulation program (Vol 2)", Journal of fashion business, vol. 19, no. 5, (2015), pp. 63-76

[15] K. J. Barng, "A Study on the Co-evolutionary appeared in the music video and media development", Asia-pacific Journal of Multimedia Services Convergent with Art, Humanities, and Sociology, vol.6, no.5, (2016), pp. 191-200.

[16] A. Cho, "To lead the next generation of IT market, VR, AR, technology-based market trends and future prospects", Seoul; CHO, Alliance, (2015), pp. 105-107.

[17] K. J. Barng, "Research on about the tools and techniques of stage make-up education media convergence", Asia-pacific Journal of Multimedia Services Convergent with Art, Humanities, and Sociology, vol. 6, no.4, (2016), pp. 439-448.

\section{Acknowledgment}

This research was supported by Basic Science Research Program through the National Research Foundation of Korea(NRF) funded by the Ministry of Science, ICT \& Future Planning(NRF-2017R1A2B4004935).

\section{Author}

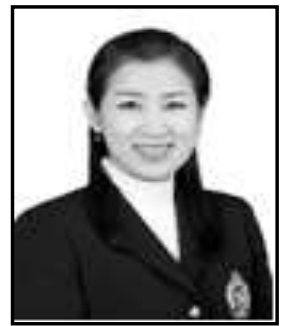

Keejung Barng, Dept. of Beauty \& Health Management, Daejeon University. 